\title{
Dental caries and obesity must be tackled together
}

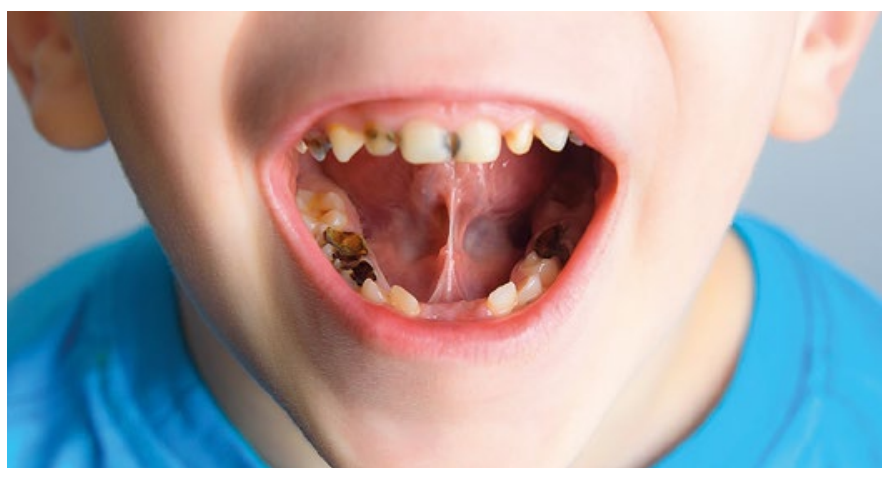

The British Society of Paediatric Dentistry (BSPD) is calling for more co-ordinated steps to tackle both dental decay and obesity, to stop thousands of children growing up to face a lifetime of disadvantage.

The BSPD has published a position statement ${ }^{1}$ on obesity and dental caries which includes a new analysis from Public Health England (PHE) showing that children who are underweight, overweight or very overweight are more likely to have experienced dental decay than those of a healthy weight. New research on sugar consumption is also included: current figures suggest children and young people consume up to three times more sugar than the recommended levels.

The statement highlights that the children who are suffering most with dental caries and obesity are from the most deprived areas, making the conditions symptomatic of widening health inequalities. Children from deprived areas of the UK are twice as likely to be obese.

The British Society of Paediatric Dentistry (BSPD) wants to see clearer food labelling and further sugar reduction in food and drinks frequently consumed by children. It supports:

- Healthy lifestyle campaigns such as those led by Change4Life in England and Wales and the 'Eat Better, Feel Better' initiative in Scotland

- Measures designed to halve the prevalence of childhood obesity by 2030 , such as the soft drinks sugar levy and restrictions on the advertisement of unhealthy food and drink, especially high sugar products.

\section{It also calls for:}

- Co-ordinated and multi-agency approaches to the management of obesity and dental decay

- An extension of the free school meal scheme to cover holiday periods for those most in need.

Originally published in 2015 , the updated 2020 position statement includes new statistics and research on obesity, dental caries and the risks of general anaesthesia for obese children.

Currently, obesity and dental decay are tackled independently by different arms of the NHS. BSPD believes there needs to be a greater willingness for all agencies to work innovatively together.

Claire Stevens, spokesperson for BSPD, said: 'In our clinics, our members see children who are obese at one end of the spectrum or underweight at the other. A poor diet is the unifying factor between their dental health and their weight. Children who have poor dental health and are obese are likely to experience a lifetime of disadvantage'.

The position statement also highlights that there are doubts about the affordability of the Eatwell Guide. The food budget of over half of UK households is likely to be insufficient to meet the Guide's recommendations, potentially increasing the risk of excess weight and dental decay.

Finally, there is increasing evidence that excess weight is associated with an increased COVID-19 risk. A report on the impact of the COVID-19 pandemic by the All-Party Group for a Fit and Healthy Childhood (2020) highlights how COVID-19 has placed further strain on inequalities, including food security and availability. ${ }^{2}$

\section{References}

1. British Society of Paediatric Dentistry. Position Statements. December 2020. Available at: https://www.bspd.co.uk/Professionals/Resources/Position-Statements (accessed December 2020)

2. APPG. The All-Party Parliamentary Group on a Fit and Healthy Childhood. October 2020 Available at: https://fhcappg.org.uk/?p=2616 (accessed December 2020).

\section{GDC highlights importance of clear public safety COVID information}

New research published on 3 December by the General Dental Council (GDC) reveals that while most people (61\%) feel just as confident about visiting the dentist as they did before the pandemic, almost a third (32\%) feel less safe, with worries about COVID safety being cited.

The research, which forms part of a wider programme of work to understand the impact of COVID-19 on dentistry and support the recovery of oral health services, also shows the potential that health inequalities are being created and exacerbated, as people from ethnic minority backgrounds and those with physical or mental health conditions were more likely to have concerns about visiting dental practices in future.

In addition, the report highlights that measures already being taken by dental practices, such as providing clear information in advance of appointments about the COVID-19 control measures that are in place, including staff wearing PPE and extra cleaning before and after appointments, have a real impact on patient confidence.

Stefan Czerniawski, Executive Director, Strategy at the GDC, said: 'The dental profession has worked tirelessly to support patients through the extraordinary difficulties resulting from this crisis. The challenge for all of us now is to ensure that patients feel confident in being able to access the dental treatment they need, and it's important that they continue to hear clear messages that it is safe to return. This research provides rich insights into the factors which influence behaviour and expectations'.

To view the full report and read about the emerging picture from the wider programme of work, visit gdc-uk.org/impactsofcovid19. 